\title{
Guest Editorial: Multimedia Applications for Smart device and Equipment
}

\author{
Tai-hoon Kim ${ }^{1} \cdot$ Sabah Mohammed ${ }^{2} \cdot \operatorname{Carlos~Ramos}^{3}$ • \\ Wai-Chi Fang ${ }^{4}$
}

(C) Springer Science+Business Media New York 2015

We are very happy to publish this special issue of MTAP published by Springer.

This issue contains 20 articles coming from various countries, among which we mention the UK, Korea, China, Taiwan, Australia, Brazil, Spain, and India. Achieving such a high quality of papers would have been impossible without the huge work that was undertaken by the Editorial Board members and external reviewers. We take this opportunity to thank them for their great support and cooperation.

In the paper "Interactive browsing of image collections on mobile devices" (10.1007/ s11042-014-1875-8), authors presented an image browsing system for interactive exploration of image collections on mobile devices. Images were arranged so that visually similar images were grouped together while large image repositories became accessible through a hierarchical, browsable tree structure, arranged on a hexagonal lattice. The developed system provided an intuitive and fast interface for navigating through image databases using a variety of touch gestures.

The paper "An adaptive loss protection for video transmission over content-centric networks" (10.1007/s11042-014-1874-9), proposed an Adaptive Loss Protection scheme for video transmission over Content-Centric Networks (ALP-CCN). The proposed ALP-CCN introduced the Forward Error Correction (FEC) mechanism with the $\mathrm{CCN}$ retransmission

Sabah Mohammed

sabah.mohammed@lakeheadu.ca

Tai-hoon Kim

taihoonn@daum.net

Carlos Ramos

csr@dei.isep.ipp.pt

Wai-Chi Fang

dr.wfang@gmail.com

1 University of Tasmania, Centenary Building, room 350, Private Bag 87, Hobart, TAS 7001, Australia

2 Lakehead University, 955 Oliver Road, Thunder Bay, Ontario P7B 5E1, Canada

3 ISEP/IPP, Rua Dr. António Bernardino de Almeida, 431, 4200-072 Porto, Portugal

4 National Chiao Tung University, 1001 University Road, Hsinchu, Taiwan 300, Republic of China 
approach. By monitoring the packet loss rate and transmission delay, their proposed ALP-CCN coordinated FEC and retransmission adaptively.

In the paper "Using homography relationship for auto-calibration in mobile smart-project device system" (10.1007/s11042-013-1783-3), authors conducted an auto-calibration based on homography relationship in mobile smart-project device system to take advantage of the capability of the projector to project an image onto any opaque and unflat plane, thus breaking through the restricted display interfaces of mobile devices. Moreover, authors also proposed the method that named FDPA (standing for Fast Detecting Projection Area) to reduce the battery power consumption via decreasing the calculation cost of the auto-calibration.

The paper "Implementation of a smartphone sensing system with social networks: a location-aware mobile application" (10.1007/s11042-013-1782-4), designed a webintegrated, cross-platform communications interface based on smartphone technology to overcome many of the difficulties inherent in visiting crowded public spaces, events, or exhibitions. The goal of this paper was to provide an alternative to the standard tour itinerary with a locationspecific, customized tour guide system that also makes use of social networks to locate friends.

In the paper "Dynamic path determination policy for distributed multimedia content adaptation" (10.1007/s11042-013-1735-y), authors proposed a dynamic multi-criteria path determination policy that selected an optimal path to the content adaptation services that best meet the user preferences and QoS requirements. The performance of the proposed approach was studied in terms of score's fairness and reliability under different variations. The results indicated that the proposed policy performs substantially better than the baseline policy.

The paper "Smart marketing in Brazilian digital TV system through a recommendation ads" (10.1007/s11042-013-1697-0), developed an application for interactive digital TV called Smart Marketing, capable of capturing viewer navigation data through both implicit and explicit means by performing customized advertising from the process of knowledge discovery. Developed from AstroTV middleware, compatible with the Brazilian specification, its application was evaluated by means of experiment that used varied user profiles, applying into the generated database the process of knowledge discovery, which used tasks of classification and grouping.

In the paper "Multimedia content adaptation service discovery mechanism" (10.1007/ s11042-013-1696-1), authors proposed a service discovery approach that considered the client device capability and the service's attributes to discover appropriate CAS while optimizing performance and functionality. The efficiency of the proposed CAS discovery protocol was studied experimentally. The results showed that the proposed discovery approach was effective in terms of discovering appropriate content adaptation services.

In order to improve the visualization simulation effect of water wave, authors used the images of actual water wave as a feedback to correct the control parameters of shallow water (SW) equations in the paper "Water wave visualization simulation using feedback of image texture analysis" (10.1007/ s11042-013-1683-6). Extensive experiment results showed that the visualization simulation effect can be improved effectively by the application of authors' texture feedback-based techniques.

Functional and security aspects of Live Interactive FramE (LIFE) were presented along with details of implementation and performance evaluation in the paper "An immersive view approach by secure interactive multimedia proof-of-concept implementation" (10.1007/ s11042-013-1682-7). Conclusions of experiments showed that LIFE enabled practical secure media streaming solution with optimal video quality settings.

The paper "A new robust and efficient multiple watermarking scheme" (10.1007/s11042013-1681-8) presented a novel multiple watermarking scheme for copyright protection and authentication. The core idea was to segment the host image into nonoverlapping blocks by the 
means of space filling curve and based on the amount of DCT energy in the blocks. The threshold values were then selected to embed multiple watermarks in different blocks.

By using the simulative method, in the paper "Simulative research on the function of internet of things basing on the changing of topological structure" (10.1007/s11042-013-1615-5), authors studied the relationship between the changes of topological structure and network performance before the network resource reset, which can improve the project of resource replacement, and, furthermore, improve the network performance.

The contribution of the paper "A hybrid construction of a decision tree for multimedia contents" (10.1007/s11042-013-1614-6) was twofold. First, authors proposed an approach for constructing a decision tree-based classification model for multimedia contents. Second, in order to speed up the performance of the proposed model, authors proposed a hybrid CPUGPU approach for construction of decision tree on graphic processing unit (GPU).

For clinicians working at a distance, the amounts of data generated by all in-home patient telematics devices posed questions on how best to intelligently filter, analyze, and interpret this data to make diagnoses and respond to changes in patient conditions. In order to manage this issue, expert systems, applied for decades in other health fields, might play a role. In the paper "Exploring a role for MCRDR in enhancing telehealth diagnostics" (10.1007/s11042-013-1613-7), authors explored how one type of expert system, Multiple Classification Ripple Down Rules (MCRDR), might address the issues.

The objective of the paper "Dynamical model for gamification of learning (DMGL)" (10. 1007/s11042-013-1612-8) was to hypothesize "Dynamical model of educational effectiveness for the gamification of learning, and to widely announce a pure and right function of game through our model." For the theoretical contribution of gamification, authors proposed a dynamical model of game-based learning that aimed to maximize educational effectiveness that correlates with the four main primary factors (curiosity, challenge, fantasy, and control).

Data clustering and analyzing techniques were studied by using hierarchical clustering method in the paper "Data clustering and analyzing techniques using hierarchical clustering method" (10.1007/s11042-013-1611-9). A matrix of words was constructed with a randomly chosen RSS list. By collecting data from this list, a matrix was built. In the matrix, each row corresponded to an article and each column represented a word. Based on the matrix, a hierarchical clustering algorithm was designed.

The main objective in the paper "Virtual learning communities: unsolved troubles" (10.1007/ s11042-013-1543-4) was to corroborate the hypothesis raised, for which the problem was described, and also to review the state of the art was made, noticing that it appeared recurrently. Besides, the degree of fitness in current LMS was valued, verifying if they possessed functionalities in their architectures to enable communities in a cooperative cloud of e-learning.

Currently, little is known about the problems that prevent the formation of the grid of Expertise in Virtual Learning Communities. Such techniques are not being implemented optimally and efficiently. In the paper "Solving technological isolation to build virtual learning communities" (10. 1007/s11042-013-1542-5), authors presented a description of these problems. This work is important for community members (directors, teachers, researchers, and practitioners) because it offers a conceptual framework that helps understand these scenarios and can provide useful design requirements when generating learning services for the community.

In the paper "Xen-based virtual honeypot system for smart device" (10.1007/s11042-0131499-4), the Xen-based virtual machine solution was proposed to build the virtual honeynet. A virtual honeynet deployed a honeynet on a physical machine based on virtual machine technology with the advantages of low cost as well as convenient management, and maintenance features. 
The paper "Web from preprocessor for crawling" (10.1007/s11042-013-1460-6) presented a novel method for searching candidate web form field values. The challenge was to map more application content than using the field value pairs included by default. Authors' method tried to get form field values executing the client side code and looking for candidate values in an external data source.

In the paper "Personalized item generation method for adaptive testing systems" (10.1007/ s11042-013-1421-0), authors used adaptive testing to estimate a learner's ability and to determine a number of learner characteristics to create a learner profile. This method selected items and created a customized assessment sheet for adaptive testing that considered both the learner's level and characteristics.

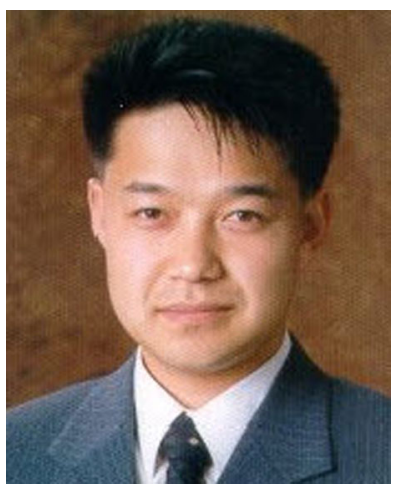

Tai-hoon Kim received his Ph.D. in School of Information and Computer Science from University of Tasmania, Australia. After working with Technical Institute of Shindoricoh as a researcher and working at the Korea Information Security Agency as a senior researcher, he worked at the DSC (Defense Security Command). After working with Hannam University four and a half year as an associate professor, now he is currently working at Sungshin W. Universty. He published about 200 papers till now.

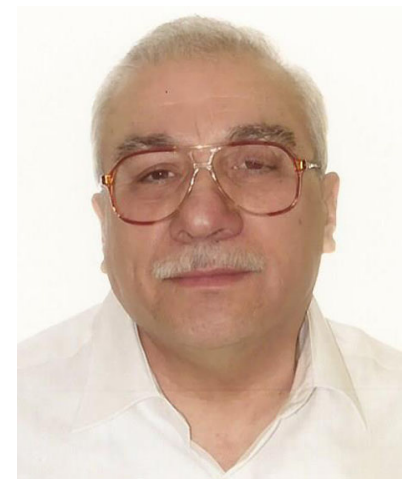

Dr. Sabah Mohammed research interest is in intelligent systems that have to operate in large, nondeterministic, cooperative, survivable, adaptive or partially known domains. Although his research is inspired by his $\mathrm{PhD}$ work back in 1981 on the employment of some Brain Activity Structures based techniques for decision making 
(planning and learning) that enable processes (e.g. agents, mobile objects) and collaborative processes to act intelligently in their environments to timely achieve the required goals. Sabah has extended his research vision to include constructivism and focus more on the nature of knowledge. Since knowledge is created by people and influenced by their values and culture, Sabah's research has been shifted more towards net centric systems (e.g. Cloud Computing, Social Networking and Enterprise Systems, Web-Based Systems,Health Informatics and Big Data). During the last ten years at Lakehead, Sabah's research is focused on developing ubiquitous healthcare systems that enable sharing securely medical knowledge and data. In particular sharing Electronic Health Records (EHRs) over the Web/cloud is one of the very challenging problems that Sabah is trying to solve. Sabah believes that finding good solutions for sharing EHRs requires approaches that cut across many different fields (e.g. Semantic Web, Web 2.0, Web 3.0, Ubiquitous Computing, Cloud Computing, Medical Informatics, $\mathrm{XML} / \mathrm{JSON}$ Security, Artificial Intelligence and Big Data). He has published several notable publications related to this hybrid area. During 2010 Sabah edited and authored a book on "Ubiquitous Health and Medical Informatics: The Ubiquity 2.0 and Beyond" by IGI Global, USA. Sabah's professional career includes many achievements. He is the Editor-in-Chief of three international journals on Big Data Security Intelligence (IJBDSI), Ubiquitous Multimedia (IJMUE) and Education\&Learning (IJEL). Sabah is also the Honarary Editor for the Journal of Emerging Technologies in Web Intelligence (JETWI) Sabah was also a Visiting Scholar at the Math and Computer Science Department, Laurentian University (Winter 2008). He holds the status of Professional Software Engineer of Ontario (P.Eng) and Canada's Information Processing Professional (ISP) as well as IEEE Senior Member. He is an active research member of Canada's e-Health Interoperability group, the coordinator on Northern Ontario Web Intelligence Research Group and a member of Ontario Partnership for Innovation and Commercialization. Besides being an active member of the Department of Computer Science here at Lakehead University, Sabah is among the core faculty members of both the Lakehead University Bioinformatics and BioTechnology programs. Moreover, Sabah is an Adjunct Research Professor with the University of Western Ontario. Sabah is also a core member of the BioTechnology $\mathrm{PhD}$ program. His teaching involves many important courses in Computer Science including: Mobile Programming, Game Programming, Artificial Intelligence, Programming Languages and Language Processors, Web Programming and Big Data. During 2005, 2006 and 2015, Sabah was the recipient of the LU Merit Awards (2006, 2015) in Research and Teaching as well as the LU Contribution to Teaching Award (2004). Sabah has also some notable administrative services as he chaired prior to his LU position three Computer Science and Information Systems departments as well as being on several notable LU Senate Committees. Sabah served on the LU Senate for the period 20102013. Moreover, Sabah has published more than 90 refereed articles, chapters in books and four textbooks. He supervised more than 40 Masters Students and one PhD student.

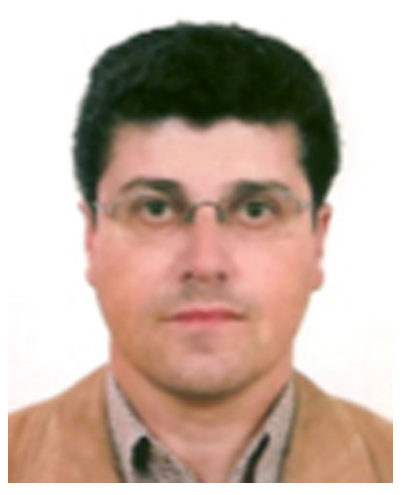

Carlos Ramos got his graduation from the University of Porto, Portugal, in 1986 and the PhD degree from the same university in 1993. Carlos Ramos has about 60 publications in scientific journals and magazines and more than 250 publications in Scientific Conferences Proceedings. Currently he is a Vice-President of the Polytechnic of Porto, the largest Polytechnic institution in Portugal, being responsible for the R\&D, Innovation and Enterpreneurship, and Internacionalization areas. 


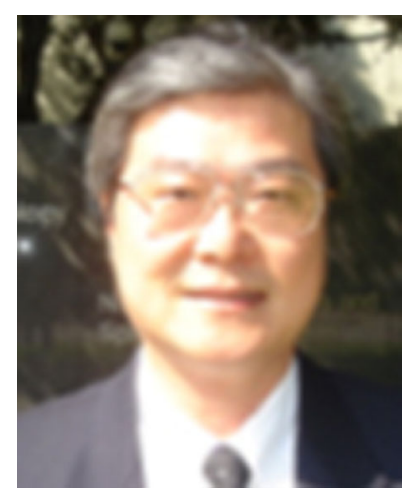

Wai Chi Fang is an IEEE Fellow and serves as the Vice President of IEEE Systems Council. He serves on the Advisory Board of IEEE Systems Journal and the Advisory Board of International Journal of Innovative Computing, Information \& Control. He was an elected Governor of the IEEE Circuits andSystems Society (2003-2008) and an AdCom member of the IEEE Nanotechnology Council. He was theChairman of IEEE CASS Technical Committee on Nanoelectronics and Gigascale Systems. 